TITLE:

\title{
Development and characterization of nuclear microsatellite markers in Aphananthe aspera (Thunb.) Planch. (Cannabaceae)
}

\section{$\operatorname{AUTHOR}(\mathrm{S}):$}

Sakagami, Taishi; Sakaguchi, Shota; Isagi, Yuji; Setoguchi, Hiroaki

\section{CITATION:}

Sakagami, Taishi ...[et al]. Development and characterization of nuclear microsatellite markers in Aphananthe aspera (Thunb.) Planch. (Cannabaceae). Journal of Forest Research 2020, 25(2): 120-123

ISSUE DATE:

2020-03

URL:

http://hdl.handle.net/2433/251018

\section{RIGHT:}

This is an Accepted Manuscript of an article published by Taylor \& Francis in Journal of Forest Research on 24 March 2020, available online: http://www.tandfonline.com/10.1080/13416979.2020.1744232;; The full-text file will be made open to the public on 24 March 2021 in accordance with publisher's 'Terms and Conditions for Self-Archiving'.; この論文 は出版社版でありません。引用の際には出版社版をご磼認ご利用ください。; This is not the published version. Please cite only the published version. 
Sakagami et al - Aphananthe SSR markers

\section{Short communication}

2 Title

3 Development and characterization of nuclear microsatellite markers in Aphananthe

4 aspera (Thunb.) Planch. (Cannabaceae)

5

\section{Authors}

$7 \quad$ Taishi Sakagami ${ }^{1}$, Shota Sakaguchi ${ }^{2,4}$, Yuji Isagi $^{3}$, Hiroaki Setoguchi ${ }^{2,4}$

\section{$9 \quad$ Affiliations}

$10 \quad{ }^{1}$ Faculty of Integrated Human Studies, Kyoto University, Kyoto, Japan; ${ }^{2}$ Graduate

11 School of Human and Environmental Studies, Kyoto University, Kyoto, Japan;

$12{ }^{3}$ Division of Forest and Biomaterials Science, Graduate School of Agriculture, Kyoto

13 University, Kyoto, Japan

15 Email addresses: TS: skgt0404@gmail.com, SS: sakaguchi.shota.6a@kyoto-u.ac.jp,

16 YI: isagiy@kais.kyoto-u.ac.jp, HS: setoguchi.hiroaki.2c@kyoto-u.ac.jp

$17 \quad{ }^{4}$ Author for $\quad$ correspondence: setoguchi.hiroaki.2c@kyoto-u.ac.jp,

18 sakaguci54@gmail.com 
Sakagami et al - Aphananthe SSR markers

21 Running head: Aphananthe SSR markers

22 Keywords: Aphananthe aspera, Cannabaceae, genetic diversity, microsatellite markers 
Sakagami et al - Aphananthe SSR markers

\section{Abstract}

24 Nuclear microsatellite markers were developed for Aphananthe aspera (Thunb.)

25 Planch. (Cannabaceae), a deciduous canopy tree species distributed in East Asia, to

26 evaluate the genetic diversity and genetic structure of $A$. aspera populations in remnant

27 forest fragments in urbanized areas of Japan. A total of 94 primer pairs were designed

28 based on genomic sequence data. Of the 25 primer pairs which showed clear

29 microsatellite peaks, 20 pairs showed allelic polymorphisms in 57 individuals collected

30 from two distant populations. The length of PCR products ranged from 120 to $482 \mathrm{bp}$,

31 and expected heterozygosity for the 20 microsatellite markers ranged from 0.017 to

32 0.768. These newly developed SSR markers will be used in population genetic studies

33 of $A$. aspera to evaluate genetic diversity and the extent of genetic isolation of the

34 fragmented populations in urban areas. 
Sakagami et al - Aphananthe SSR markers

\section{Introduction}

Habitat fragmentation can have major genetic and demographic consequences on populations (Honnay et al. 1999; Oostermeijer et al. 2003; Fahrig 2017). It is predicted that reduction in effective population size and the decreased population connectivity can lead to negative effects such as greater inbreeding (Keller and Waller 2002), restricted gene flow (Browne and Karubian 2018), and reduced immigration rates (Couvet 2002; Dubreuil et al. 2010). While the effects of habitat fragmentation on tree species may be buffered by their longevity and greater capabilities of gene dispersals, empirical studies showed the consequences vary by species and context (Lowe et al. 2015), requiring further examinations in different study systems.

Aphananthe aspera (Thunb.) (Cannabaceae) is a temperate broad-leaved deciduous tree species widely distributed in Japan (from subtropical Okinawa Island to temperate Kanto region), extending to the Korean peninsula, China, and Taiwan. It is a long-lived (in some cases up to $>500$ years) and wind-pollinated canopy tree. Ripe berries of $A$. aspera are black purple in color in autumn, and mainly dispersed by birds (Yoshikawa and Kikuzawa 2009). It sometimes becomes dominant in warm-temperate forests in alluvial lowlands, but currently forms small, scattered populations due to human activities especially in urbanized area in Japan (Kimura et al. 2019). Although 
Sakagami et al - Aphananthe SSR markers

the species is ecologically important in terms of dominance and interaction with avian and animal species in warm-temperate forests, there have been no studies on the species' genetic diversity and genetic differentiation of the fragmented $A$. aspera populations. Microsatellite markers (or simple sequence repeat, SSR) are highly polymorphic, codominant, and useful to evaluate population genetic characteristics (Jones and Ardren 2003). To investigate the negative effects on gene diversity and genetic structure by fragmentation, microsatellite markers that can be applied to $A$. aspera are needed. In this study, we developed nuclear microsatellite markers from $A$. aspera using genomic sequence data and evaluated their polymorphisms.

\section{Materials and Methods}

We assembled the genomic sequence reads of $A$. aspera (GenBank accession numbers of raw sequence reads; Bioproject: PRJDB5250, Submission: DRA005224, Experiment: DRX069505 and Sample: DRS055811) (Sakaguchi et al. 2017), using

CLC Genomics Workbench 7.5.1 (CLC bio, Aarhus, Denmark) with the parameter settings of word size $=19$, bubble size $=161$, and minimum contig length $=100$. Then the contigs including microsatellite regions, for $\geq 6$ tri-nucleotide repeats, $\geq 8$ di-nucleotide repeats and $\geq 15$ mono-nucleotide repeats, were searched with 
Sakagami et al - Aphananthe SSR markers

MSATCOMMANDER (Faircloth 2008). In total, 4,220 microsatellite motifs were found, and 94 of them were selected to design primer pairs using Primer 3 (Rozen and Skaletsky 2000). For all the primer pairs, the forward primers were synthesized with one of four different universal sequences (5'-CACGACGTTGTAAAACGAC3', 5’-TGTGGAATTGTGAGCGG-3’, tagged with PIG-tail sequences (5'-GTTTCTT-3’) (Brownstein et al. 1996). BLASTN search for each contig was performed with a threshold of E-05 for significant hit. 57 individuals of $A$. aspera from each of the two populations [29 individuals from Shimogamo Shrine, Kyoto, Japan $\left(35^{\circ} 02^{\prime} \mathrm{N} / 135^{\circ} 46^{\prime} \mathrm{E}\right), 28$ individuals from

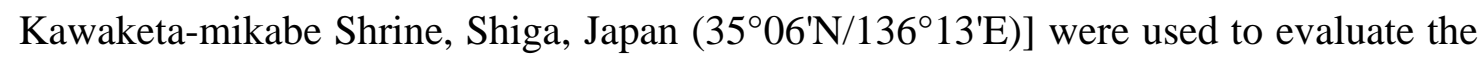
polymorphisms of the target microsatellite loci. All sampled leaves were dried in silica gel, polysaccharides removed with HEPES buffer (pH 8.0) (Setoguchi and Ohba 1995), and then genomic DNA was isolated using the CTAB method (Doyle and Doyle 1987). The PCR reaction was performed in a $10 \mu \mathrm{L}$ reaction volume containing 10-20 ng of DNA, $5 \mu \mathrm{L}$ of Multiplex PCR Master Mix (Qiagen, Hilden, Germany), $0.01 \mu \mathrm{M}$ of forward primer, $0.2 \mu \mathrm{M}$ of reverse primer and $0.1 \mu \mathrm{M}$ of fluorescently labeled M13 primer. PCR amplification for all primer pairs started with 3 min at $95^{\circ} \mathrm{C}$ for initial 
Sakagami et al - Aphananthe SSR markers

89

90

91

denaturation, 35 cycles of denaturation at $95^{\circ} \mathrm{C}$ for $30 \mathrm{~s}$, primer annealing at $60^{\circ} \mathrm{C}$ for 3 min, and extension at $68^{\circ} \mathrm{C}$ for $1 \mathrm{~min}$, and a final extension for $20 \mathrm{~min}$ at $68^{\circ} \mathrm{C}$. PCR product was loaded onto an auto sequencer (3130 Genetic Analyzer; Applied Biosystems, Carlsbad, California, USA) to assess fragment lengths using GeneMapper software (Applied Biosystems). To evaluate the utility of developed genetic markers, genetic diversity indices (number of alleles, observed heterozygosity and expected heterozygosity) were calculated by using GenAlEx ver. 6.5.2 (Peakall and Smouse 2012). Frequency of null alleles were estimated for each combination of locus and population using CERVUS 3.0 (Marshall et al. 1998). Significance of Hardy-Weinberg equilibrium and linkage disequilibrium between loci were tested by GenAlEx ver. 6.5.2.

\section{Results and discussion}

Of 94 primer pairs tested, 25 pairs showed clear microsatellite peaks (Table 1) and 20 pairs displayed allelic polymorphisms in the two populations of A. aspera (Table 2). The corresponding sequences of these regions were deposited in DDBJ Center: accession numbers LC360729- LC360744 (Table 1). In the populations of $A$. aspera, the number of alleles per locus ranged from 1 to 8 , the observed heterozygosity 
Sakagami et al - Aphananthe SSR markers

107 ranged from 0.000 to 0.724 and the expected heterozygosity ranged from 0.000 to

1080.783 (Table 2). One locus (Aa_105260) showed consistently significant deviations

109 from Hardy-Weinberg equilibrium in both populations $(P<0.001)$, and relatively high

110 frequencies of null alleles were estimated ( 0.563 for Shimogamo Shrine and 0.673 for

111 Kawaketa-mikabe Shrine, respectively). Hence, care must be taken when applying the

112 marker (Aa_105260) in any future genetic research. No locus pairs showed significant

113 linkage disequilibrium $(P>0.05)$. In conclusion, the novel 20 nuclear microsatellite

114 markers were polymorphic and reliably scorable in A. aspera. These markers will be

115 useful in future studies investigating the population genetics, and evolutionary history

116 and genetic interactions within populations of the species.

117

\section{Acknowledgements}

We are grateful to S. Mori, R. Nakamasu for collecting plant materials, and to

two anonymous reviewers and coordinating editor for their useful comments on this

manuscript Research permissions were provided by Shimogamo Shrine and

122 Kawaketa-mikabe Shrine. This research was supported by the Japan Society for the

123 Promotion of Science Grant-in-Aid for Scientific Research (JSPS KAKENHI

124 16H04827, 16H04831 and 25291085) and the Environmental Research and

125 Technology Development Fund of the Ministry of the Environment (4-1702, 4-1902), 
Sakagami et al - Aphananthe SSR markers

126 and SICORP Program of the Japan Science and Technology Agency (grant no.

127 4-1403).

128 
Sakagami et al - Aphananthe SSR markers

129

130

131

132

133

134

135

136

137

138

139

140

141

\section{References}

Blacket M, Robin C, Good R, Lee S, Miller A (2012) Universal primers for fluorescent labelling of PCR fragments-an efficient and cost-effective approach to genotyping by fluorescence. Molecular ecology resources 12:456-463

Browne L, Karubian J (2018) Habitat loss and fragmentation reduce effective gene flow by disrupting seed dispersal in a neotropical palm. Mol Ecol 27:3055-3069

Brownstein MJ, Carpten JD, Smith JR (1996) Modulation of non-templated nucleotide addition by taq DNA polymerase: Primer modifications that facilitate genotyping. Biotechniques 20:1004-+

Couvet D (2002) Deleterious effects of restricted gene flow in fragmented populations. Conservation Biology 16:369-376

Doyle JJ, Doyle JL (1987) A rapid DNA isolation procedure for small quantities of fresh leaf tissue. Phytochem. Bull Bot Soc Am 19

Dubreuil M, Riba M, González-Martínez SC, Vendramin GG, Sebastiani F, Mayol M (2010) Genetic effects of chronic habitat fragmentation revisited: Strong genetic structure in a temperate tree, Taxus baccata (Taxaceae), with great dispersal capability. American Journal of Botany 97:303-310 
Sakagami et al - Aphananthe SSR markers

147 Fahrig L (2017) Ecological Responses to Habitat Fragmentation Per Se. Annual Review of Ecology, Evolution, and Systematics 48:1-23

149 Faircloth BC (2008) MSATCOMMANDER: detection of microsatellite repeat arrays and automated, locus-specific primer design. Molecular Ecology Resources 8:92-94

Honnay O, Hermy M, Coppin P (1999) Effects of area, age and diversity of forest patches in Belgium on plant species richness, and implications for conservation and reforestation. Biological Conservation 87:73-84

Jones AG, Ardren WR (2003) Methods of parentage analysis in natural populations. Molecular Ecology 12:2511-2523

Keller LF, Waller DM (2002) Inbreeding effects in wild populations. Trends in Ecology \& Evolution 17:230-241

Kimura M, Imanishi J, Fukamachi K, Sakamoto K, Shibata S (2019) Remnant pattern of woods and scattered trees of Celtis sinensis, Aphananthe aspera and Zelkova Institute of Landscape Architecture 82:697-702 forest fragmentation genetics — no longer a paradox-we were just looking in 
Sakagami et al - Aphananthe SSR markers the wrong place. Heredity 115:97

Marshall T, Slate J, Kruuk L, Pemberton J (1998) Statistical confidence for likelihood-based paternity inference in natural populations. Molecular ecology 7:639-655

Oostermeijer J, Luijten S, Den Nijs J (2003) Integrating demographic and genetic approaches in plant conservation. Biological Conservation 113:389-398

Peakall R, Smouse PE (2012) GenAlEx 6.5: genetic analysis in Excel. Population genetic software for teaching and research-an update. Bioinformatics 28:2537-2539

Rozen S, Skaletsky HJ (2000) Primer3 on the www for general users and for biologist programmers. In: Krawetz S, Misener S (eds) Bioinformatics Methods and Protocols: Methods in Molecular Biology. Humana Press, Totowa, NJ

Sakaguchi S, Ueno S, Tsumura Y, Setoguchi H, Ito M, Hattori C, Nozoe S, Takahashi D, Nakamasu R, Sakagami T, Lannuzel G, Fogliani B, Wulff AS, L'Huillier L, Isagi Y (2017) Application of a simplified method of chloroplast enrichment to small amounts of tissue for chloroplast genome sequencing. Applications in Plant Sciences 5:1700002

Setoguchi H, Ohba H (1995) Phylogenetic relationships in Crossostylis 
Sakagami et al - Aphananthe SSR markers

Yoshikawa T, Kikuzawa K (2009) Pre-dispersal Seed Predation by a Granivorous Bird, 
Sakagami et al - Aphananthe SSR markers

\section{Table 1}

191 Nuclear microsatellite markers for Aphananthe aspera (Thunb.) Planch.. Shown for each primer pair are repeat motif, forward and reverse 192 primer sequence (with tag sequence), the results of BLASTN database search with E-value and the accession number in DDBJ, and allele 193 size range.

\begin{tabular}{|c|c|c|c|c|c|c|}
\hline Locus & $\begin{array}{l}\text { Repeat } \\
\text { motif }\end{array}$ & Sequence & BLASTN & E-value & $\begin{array}{l}\text { GenBank } \\
\text { Accession } \\
\text { No. }\end{array}$ & $\begin{array}{l}\text { Allele } \\
\text { size } \\
\text { range } \\
\text { (bp) }\end{array}$ \\
\hline Aa_115573 & $(\mathrm{GT})_{12}$ & $\begin{array}{l}\text { F:CACGACGTTGTAAAACGACTAATGGAGCCGCATCACTTG } \\
\text { R:GTTTCTTACAGCTAGCTATTTCGTGGAAG }\end{array}$ & No significant hits & - & LC360719 & $122-128$ \\
\hline Аа_84574 & $(\mathrm{AAT})_{10}$ & $\begin{array}{l}\text { F:CGGAGAGCCGAGAGGTGTCGGCACCTTACACATTCTTG } \\
\text { R:GTTTCTTCGTTAAGATGGGCAGACAGG }\end{array}$ & No significant hits & - & LC360720 & $171-180$ \\
\hline Aa_105260 & $(\mathrm{AC})_{14}$ & $\begin{array}{l}\text { F:CACGACGTTGTAAAACGACAAATTCACAGGAGCACATTTGG } \\
\text { R:GTTTCTTGCTGCATGGCCATCTAACAG }\end{array}$ & No significant hits & - & LC360721 & $200-224$ \\
\hline Aa_96067 & $(\mathrm{AG})_{12}$ & $\begin{array}{l}\text { F:CTATAGGGCACGCGTGGTCGGGCTCGTTTGAAGAAGAC } \\
\text { R:GTTTCTTGCCGTTGACTCAGACTACCC }\end{array}$ & No significant hits & - & LC360722 & $250-262$ \\
\hline Aa_103887 & $(\mathrm{GA})_{11}$ & $\begin{array}{l}\text { F:CACGACGTTGTAAAACGACTGCGGGTGAGAGATTCTAGC } \\
\text { R:GTTTCTTGGAAGGTCAAAGCCCAAAGG }\end{array}$ & No significant hits & - & LC360723 & $356-366$ \\
\hline Аа_95198 & $(\mathrm{TG})_{13}$ & $\begin{array}{l}\text { F:CGGAGAGCCGAGAGGTGCCACGTCCCACTAGCTTCTC } \\
\text { R:GTTTCTTGAACATGGCCGTGGTGTAG }\end{array}$ & No significant hits & - & LC360724 & $425-437$ \\
\hline
\end{tabular}


Sakagami et al - Aphananthe SSR markers

\begin{tabular}{|c|c|c|c|c|c|c|}
\hline Aa_119647 & $(\mathrm{GA})_{10}$ & $\begin{array}{l}\text { F:TGTGGAATTGTGAGCGGGGCTGTCTTAAGATGGGAGAAG } \\
\text { R:GTTTCTTGTATTACAGCCCTGCACGAC }\end{array}$ & $\begin{array}{l}\text { Vitis vinifera, whole genome shotgun sequence, } \\
\text { contig VV78X118074.3, clone ENTAV } 115\end{array}$ & $2.00 \mathrm{E}-12$ & LC360725 & $120-144$ \\
\hline Aa_10258 & $(\mathrm{TCT})_{27}$ & $\begin{array}{l}\text { F:TGTGGAATTGTGAGCGGTGGCCAGAAGTACCCTTGTC } \\
\text { R:GTTTCTTCTCCTCGTCCGTATCCTTGG }\end{array}$ & No significant hits & - & LC360726 & 421 \\
\hline Aa_27760 & $(\mathrm{CA})_{11}$ & $\begin{array}{l}\text { F:CGGAGAGCCGAGAGGTGACACACCGGAAGAAGAAAGC } \\
\text { R:GTTTCTTGGACCACCTGCATACAAGAG }\end{array}$ & $\begin{array}{l}\text { PREDICTED: Ziziphus jujuba myb-related protein } \\
\text { Myb4-like (LOC107416961), mRNA }\end{array}$ & $2.00 \mathrm{E}-15$ & LC360727 & $460-482$ \\
\hline Aa_105963 & $(\mathrm{GA})_{12}$ & $\begin{array}{l}\text { F:CTATAGGGCACGCGTGGTACTTTGCACAGATGAACAGAAC } \\
\text { R:GTTTCTTTATTGCTGCTGGAGGATGGC }\end{array}$ & $\begin{array}{l}\text { PREDICTED: Prunus avium ultraviolet-B receptor } \\
\text { UVR8 (LOC110758246), mRNA }\end{array}$ & $1.00 \mathrm{E}-14$ & LC360728 & $127-143$ \\
\hline Aa_104223 & $(\mathrm{GT})_{9}$ & $\begin{array}{l}\text { F:TGTGGAATTGTGAGCGGTGGGAATTTCAAATCCTGGCAG } \\
\text { R:GTTTCTTACAAGAAGAGTCAAGCGCAG }\end{array}$ & No significant hits & - & LC360729 & $187-197$ \\
\hline Aa_50376 & $(\mathrm{TC})_{11}$ & $\begin{array}{l}\text { F:CTATAGGGCACGCGTGGTGGTGAACTTGTTGGGAGCAC } \\
\text { R:GTTTCTTCATCCCACCCAATTTCCACC }\end{array}$ & No significant hits & - & LC360730 & $225-231$ \\
\hline Aa_104956 & $(\mathrm{GA}) 9$ & $\begin{array}{l}\text { F:CACGACGTTGTAAAACGACGTCCTTGTCACGTGGCTTTC } \\
\text { R:GTTTCTTTTTGCCAGAGATTGCAATTGG }\end{array}$ & No significant hits & - & LC360731 & $240-276$ \\
\hline Aa_44791 & $(\mathrm{AC})_{10}$ & $\begin{array}{l}\text { F:CACGACGTTGTAAAACGACTCGTAGGAATTTCGCACGTG } \\
\text { R:GTTTCTTTCAGGATAATGGTGTCAAGCG }\end{array}$ & No significant hits & - & LC360732 & $423-439$ \\
\hline Aa_104111 & $(\mathrm{AG})_{11}$ & $\begin{array}{l}\text { F:CTATAGGGCACGCGTGGTACCAAACTCAACCACACCAG } \\
\text { R:GTTTCTTGAAAGGATTGGCGTCGTTCC }\end{array}$ & No significant hits & - & LC360733 & $444-460$ \\
\hline Aa_10240 & $(\mathrm{TC})_{11}$ & $\begin{array}{l}\text { F:CGGAGAGCCGAGAGGTGAGTTTGTTTCCTCTTCTTTCTG } \\
\text { R:GTTTCTTGTCATTATTGCCTAAGTTGCCC }\end{array}$ & No significant hits & - & LC360734 & $145-159$ \\
\hline Aa_13484 & $(\mathrm{AG})_{12}$ & F:CTATAGGGCACGCGTGGTGATAAGGCGGGAGGAGTACG & No significant hits & - & LC360735 & 193-205 \\
\hline
\end{tabular}


Sakagami et al - Aphananthe SSR markers

R:GTTTCTTCCCATTTGCCCGGTTCTTTC

Aa_13178 $(\mathrm{AGT})_{7}$ F:CGGAGAGCCGAGAGGTGAAAGAAGGTTGAAGGCTGCG

Aa_12869

Aa_13711 (AAT)6 F:TGTGGAATTGTGAGCGGATTGTGGGCCTCGACCTTAG

Aa_19942 (A) $)_{18}$ F:CGGAGAGCCGAGAGGTGAGGGTGAGCTGTCCTGTAAG

Aa_10150

Aa_4179

Aa_15273

195

196

197 R:GTTTCTTTCGTAATGTTACCTCGCTAAGC R:GTTTCTTAGATCATCTTCTAATTCGCCAC

(T) $)_{18}$ F:CTATAGGGCACGCGTGGTGCTAGGTCAAACTATGGGCC R:GTTTCTTTTTCCCTCGACGAGCTATGG R:GTTTCTTAGCACCCAGTAACATCATGTG R:GTTTCTTTGAGATGGTTCGGAGGTCTG

No significant hits

No significant hits

No significant hits

No significant hits

No significant hits

No significant hits

(ATA) 7 F:CGGAGAGCCGAGAGGTGGGCTGGATTGTTTGCATTGC

R:GTTTCTTTACGAACAGAGACGTGGTGG

$($ AAT) 6 F:CACGACGTTGTAAAACGACCCCAGATTAGCTAGATGTCAC R:GTTTCTTAACCAGCAATCCGAGCATTC

(GA)9 F:CTATAGGGCACGCGTGGTACCAGGCCGATGAGAGTTTC R:GTTTCTTGGCCCGTCATTTAACCAAGG

\section{No significant hits}

Cucumis melo genomic chromosome, chr_1

No significant hits

-

LC360736

250

LC360737 187-199

LC360738

221

LC360739 327

LC360740 195-196

LC360741 234-273

2.00E-16 LC360743 191-197

- $\quad$ LC360744 244-246 
Sakagami et al - Aphananthe SSR markers

\section{Table 2}

Genetic diversity statistics for two populations of Aphananthe aspera (Thunb.) Planch. based on the newly developed polymorphic genomic microsatellite markers. Abbreviations are as follow; $N_{\mathrm{A}}$ : number of alleles, $H_{\mathrm{O}}$ : observed heterozygosity, $H_{\mathrm{E}}$ : expected heterozygosity, Null: null allele frequency estimate. Deviations from Hardy-Weinberg equilibrium are noted by asterisks ( $\left.{ }^{* *} P<0.001\right)$.

\begin{tabular}{|c|c|c|c|c|c|c|c|c|c|c|c|}
\hline \multirow[t]{2}{*}{ Locus } & \multicolumn{4}{|c|}{$\begin{array}{l}\text { Shimogamo Shrine } \\
\qquad(\mathrm{n}=29)\end{array}$} & \multicolumn{4}{|c|}{$\begin{array}{l}\text { Kawaketa-mikabe Shrine } \\
\qquad(\mathrm{n}=28)\end{array}$} & \multicolumn{3}{|c|}{$\begin{array}{c}\text { Total } \\
(\mathrm{n}=57)\end{array}$} \\
\hline & $N_{\mathrm{A}}$ & $H_{\mathrm{O}}$ & $H_{\mathrm{E}}$ & Null & $N_{\mathrm{A}}$ & $H_{\mathrm{O}}$ & $H_{\mathrm{E}}$ & Null & $N_{\mathrm{A}}$ & $H_{\mathrm{O}}$ & $H_{\mathrm{E}}$ \\
\hline Aa_4179 & 2 & 0.034 & 0.034 & -0.002 & 1 & 0.000 & 0.000 & -0.002 & 2 & 0.018 & 0.017 \\
\hline Aa_10150 & 5 & 0.556 & 0.719 & 0.201 & 5 & 0.704 & 0.783 & 0.125 & 6 & $0.630^{* *}$ & 0.768 \\
\hline Aa_10240 & 4 & 0.586 & 0.612 & 0.050 & 4 & 0.714 & 0.631 & 0.050 & 4 & 0.649 & 0.647 \\
\hline Aa_13178 & 3 & 0.241 & 0.216 & -0.060 & 2 & 0.500 & 0.497 & -0.060 & 3 & 0.368 & 0.416 \\
\hline Aa_13484 & 4 & 0.483 & 0.594 & 0.141 & 4 & 0.607 & 0.578 & 0.141 & 4 & 0.544 & 0.606 \\
\hline Aa_19942 & 2 & 0.448 & 0.348 & -0.274 & 2 & 0.464 & 0.357 & -0.286 & 2 & 0.456 & 0.352 \\
\hline Aa_27760 & 4 & 0.655 & 0.631 & -0.007 & 4 & 0.714 & 0.682 & -0.007 & 4 & 0.684 & 0.663 \\
\hline Aa_44791 & 3 & 0.500 & 0.498 & 0.002 & 2 & 0.464 & 0.469 & 0.002 & 3 & 0.482 & 0.508 \\
\hline Aa_50376 & 3 & 0.172 & 0.161 & -0.038 & 3 & 0.286 & 0.283 & -0.038 & 3 & 0.228 & 0.223 \\
\hline Aa_84574 & 3 & 0.552 & 0.565 & 0.024 & 2 & 0.393 & 0.392 & 0.024 & 3 & 0.474 & 0.492 \\
\hline Aa_95198 & 4 & 0.448 & 0.521 & 0.057 & 3 & 0.571 & 0.579 & 0.057 & 4 & 0.509 & 0.554 \\
\hline Aa_96067 & 4 & 0.483 & 0.508 & 0.037 & 4 & 0.679 & 0.658 & 0.037 & 4 & 0.579 & 0.604 \\
\hline Aa_103887 & 4 & 0.310 & 0.278 & -0.068 & 3 & 0.464 & 0.424 & -0.068 & 4 & 0.386 & 0.357 \\
\hline Aa_104111 & 5 & 0.724 & 0.736 & 0.001 & 5 & 0.679 & 0.642 & 0.001 & 5 & 0.702 & 0.699 \\
\hline Aa_104223 & 3 & 0.379 & 0.355 & -0.025 & 2 & 0.143 & 0.133 & -0.026 & 3 & 0.263 & 0.263 \\
\hline Aa_104956 & 8 & 0.621 & 0.737 & 0.116 & 4 & 0.643 & 0.600 & 0.116 & 8 & 0.632 & 0.695 \\
\hline Aa_105260 & 7 & $0.345^{* *}$ & 0.675 & 0.563 & 4 & $0.071^{* *}$ & 0.226 & 0.673 & 7 & $0.211^{* *}$ & 0.495 \\
\hline Aa_105963 & 3 & 0.517 & 0.450 & -0.043 & 3 & 0.393 & 0.456 & -0.043 & 3 & 0.456 & 0.453 \\
\hline Aa_115573 & 3 & 0.690 & 0.651 & -0.019 & 3 & 0.607 & 0.548 & -0.019 & 3 & 0.649 & 0.614 \\
\hline Aa_119647 & 3 & 0.414 & 0.424 & 0.044 & 3 & 0.536 & 0.506 & 0.044 & 4 & 0.474 & 0.474 \\
\hline Average & 3.9 & 0.458 & 0.486 & 0.035 & 3.2 & 0.482 & 0.472 & 0.036 & 4.0 & 0.470 & 0.495 \\
\hline
\end{tabular}


Sakagami et al - Aphananthe SSR markers 